ORIGINAL PAGE IS

IN-67-CR

OF POOR QUALITY

189852

\title{
Inflation in Anisotropic Scalar-Tensor Theories
}

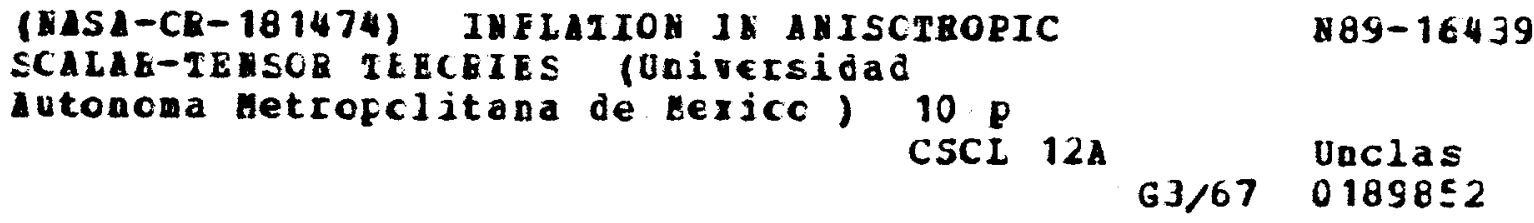

\section{Luis O. Pimentel}

\author{
Departamento de Física, \\ Universidad Autonoma Metropolitana, Iztapalapa, \\ P.O.Box 55-534, \\ C.P. 09340, México, D.F. \\ and
} Jaime Stein-Schabes

NASA/Fermilab Astrophysics Center Fermi National Accelerator Laboratory P.O. Box 500, Batavia, Il. 60510 USA

\begin{abstract}
The existence of an inflationary phase in anisotropic Scalar-Tensor Theories is investigated by means of a conformal transformation that allows us to rewrite theses theories as gravity minimally coupled to a scalar field with a a non-trivial potential. We then use the explicit form of the potential and the No Hair Theorem to conclude that there is an inflationary phase in all open or flat anisotropic spacetimes in these theories. Several examples are constructed where the effect becomes manifest.
\end{abstract}


ORIGINAL FATE IS

OF. POOR QUALITY

\section{Introduction}

The existence of a very large dimensionless number of order $10^{40}$, like the ratio of the gravitational to electrostatic forces between a proton and an electron, or the Hubble length $\left(H^{-1}\right)$ and the classical radius of the electron impressed Dirac $[1,2]$ so much that he postulated these relations as fundamental in Nature. In order to maintain these ratios fixed in time, and since the Hubble length changes as the Universe expands, he was forced to assume the Gravitational Constant $G$ was not a constant but a function of time. Moreover, it had to be inversely proportional to the age of the Universe. However, he did not provide a working theory to do this. Partly motivated by Dirac's ideas and the extra dimensionality of spacetime proposed by Kaluza, Jordan [3] constructed a theory capable of incorporating a varying $G$. The idea was to add an extra scalar degree of freedom to the already existing tensor degrees of freedom of the gravitational field and somehow interpret this scalar field as the Gravitational Constant $G$. However, the couplings where prescribed by the consistency of the theory with the constancy of the ratios as well as the principle of Equivalence (see Weinberg [4] for some more comments on this point). More recently, Brans and Dicke [5], trying to incorporate Mach's principle to a theory of gravity obtained a Scalar-Tensor Theories (STT) that is a particular case of Jordan's theory [5].

The standard cosmological model provides us with an accurate picture of the universe, however, in spite of its success and amazing flexibility, it is incapable of explaining things like the observed large scale flatness, homogeneity and isotropy of the Universe, or solving the now famous horizon and primordial monopole problems [6]. This set of affairs motivated the advent of the inflationary models first proposed by Guth and subsequently modified by Albrecht and Steinhardt and by Linde [6] independently. In the new inflationary models, the rapid expansion appears when the dynamics of the universe is dominated by the vacuum energy. This is equivalent to having a period when the energy momentum tensor driving the expansion of the universe behaves like a cosmological con- 
stant and the scale factor grows exponentially in time in a de Sitter fashion. During this time the volume increases by at least $65-\mathrm{e}$-folds. The effect of this expansion is to dilute the number of monopoles or any other topological beast created during the phase transition. Moreover, it also isotropises and homogenises the inflating region leaving behind a homogeneous, isotropic and nearly flat universe. But where does this vacuum energy comes from ? Well, it is introduce ad hoc in the form of a scalar field that has a very special potential, namely, a flat potential. It is the flat region in this potential that acts as a temporary cosmological constant.

One of the attractions of the STT, is the fact that the scalar field is inbuild in them, so its natural to ask whether inflation takes place in such theories. The question has been considered before by several authors[7], however, its been answered only in a very few restricted cases where special assumptions about the spacetime metric have been made. In most cases, it has been done by explicitly displaying an exact solution of Einstein's equations with an exponential growth. In this letter we will address the same question but in a way that allows us to obtain results for a wide class of spacetimes, all anisotropic and homogeneous models. We do this by the use of a conformal transformation that transforms the original theory with its non-linear and non-canonical couplings to gravity into one that has minimal coupling. Once we have achieved this we invoke the No Hair Theorem to show that for a large class of potentials, the transformed theory satisfies the requirements of the theorem, and so conclude that it will undergo a period of inflation. We will finish with a few examples.

\section{The Scalar-Tensor Theory}

The equations of motion for the most general STT in vacuum can be obtained from varying the following action (see Will in ref.[7]) 


$$
S=\frac{1}{16 \pi G} \int d^{4} x \sqrt{-\hat{g}}\left[\left(\hat{\phi} \hat{R}-(\hat{\phi})^{-1} \omega(\hat{\phi}) \hat{g}^{\mu \nu} \partial_{\mu} \hat{\phi} \partial_{\nu} \hat{\phi}+2 \hat{\phi} \lambda(\hat{\phi})\right]\right.
$$

where the signature is $(-,+,+,+), \omega(\hat{\phi})$ and $\lambda(\hat{\phi})$ are arbitrary functions that distinguish between different theories. $\lambda(\hat{\phi})$ is the potential function and plays a similar role to the cosmological constant. For the Brans-Dicke theory $\omega(\hat{\phi})=\omega_{0}$ and $\lambda(\hat{\phi})=0$, for the corresponding functions in other theories see ref. [5].

We shall now show that by the use of a conformal transformation it is possible to recast any STT as described above, where the coupling between the scalar field and the curvature is non-minimal and the kinetic term for $\hat{\phi}$ is not canonical, into one where the coupling is minimal and the kinetic term is canonical. The use of the conformal transformation can make the complicated problem of analyzing a coupled system, much simpler. We shall show that the resulting theory is GR plus a scalar field. Once this is achieved we shall proceed to study the existence of an inflationary phase for the universe. In most cases it reduces to an inspection of the potential, initial conditions and a qualitative description of the scalar field dynamics. We shall prove that for some STT, i.e. with special choices of $\omega(\hat{\phi})$ and $\lambda(\hat{\phi})$, will undergo inflation provided the initial conditions are correct. This will be done for any homogeneous and anisotropic model with the help of the No Hair Theorem [9]. Similar techniques have been used in [8] to prove and graphically understand similar results obtained in a variety of theories amongst which we can name the high derivative gravity $\left(R+\epsilon R^{2}\right)$, the induced gravity model $\left(\xi \phi^{2} R\right)$ and various combinations of these models. The technique has proven very powerful in predicting inflation in all these theories when the spacetime is anisotropic without having to solve explicitly any equation of motion.

Let us now make the conformal transformation $\hat{g}_{\alpha \beta} \rightarrow \Omega^{2} g_{\alpha \beta}$ and introduce a new field $\psi$ defined in the following way:

$$
\Omega \equiv \frac{1}{\sqrt{\hat{\phi}}}
$$


CRIGINE Fitis bo

OF. POOR QUALITY

$$
\psi \equiv \sqrt{8} \int^{\Omega} \frac{\sqrt{\omega}}{\Omega^{\prime}} d \Omega^{\prime}
$$

Then the action becomes

$$
S=\frac{1}{16 \pi G} \int d^{4} x \sqrt{-g}\left[R-\frac{1}{2} g^{\mu \nu} \partial_{\mu} \psi \partial_{\nu} \psi-V(\psi)\right]
$$

with $V(\psi)=-2 \Omega^{2} \lambda$ Eqn. (2.4) simply describes gravity minimally coupled to a scalar field $\psi$ with potential $V(\psi)$. It is at this stage where we need to ask for the form of the potential. If it contains a flat plateau (cosmological constant) for small or large values of the field, and if we can argue on physical grounds that the initial conditions are such that the field starts in either extreme of the potential, then the slow rollover mechanism will make it inflate. Both of these are necessary conditions for the theorem. Alternatively we need to look at the energy-momentum tensor for the scalar field and ask whether it satisfies the strong and dominant energy conditions necessary for the applicability of the No Hair Theorem (see Wald and Jensen and Stein-Schabes in [9] for more details). If so then this is an inflating model, regardless of the anisotropy present on the initial hypersurface.

Due to the generality of the STT we cannot in general say whether there is inflation or not. In order to do so we have to specialize the theory, or say something about $V(\psi)$, i.e. $\lambda(\hat{\phi})$ and $\omega(\hat{\phi})$.

\section{Examples}

In this section we shall make some special choices of $\omega(\hat{\phi})$ and $\lambda(\hat{\phi})$ and use the No Hair Theorem to show the existence of the inflationary phase.

a) $\omega(\hat{\phi})=\omega_{0}$ and $\lambda(\hat{\phi})=\sum_{n=1}^{p} a_{n} \hat{\phi}^{n} ; a_{p}<0$. Using Eqns.(2.2) and (2.3) we obtain

$$
\Omega=e^{\frac{t}{\sqrt{000}}}
$$


while the effective potential for the $\psi$ field is

$$
V(\psi)=-2 \sum_{n=1}^{p} a_{n} \Omega^{2(1-n)}=-2 \sum_{n=1}^{p} a_{n} e^{\frac{2(1-n) \psi}{\sqrt{\delta \omega}}}
$$

Under the assumption on the sign of the constant $a_{p}$ we notice that when $\psi \rightarrow \infty$ the potential goes to $2 a_{0}$, and when $\psi \rightarrow-\infty$ the potential goes to $\infty$ (see fig. 1) this is rather similar to what was found in ref. [9]. Clearly, for large $\psi$ the dynamics are dominated by the potential energy and the universe behaves as dominated by a cosmological constant. If $\psi$ is large and negative, then the model undergoes power law inflation. We do not know what the initial value of the field was, however, in the naive models we would expect it to be either very large or very small. It is clear that if initially it sits at the minimum of the potential then there will be no inflation. This example contains the original Brans-Dicke model with a non-zero cosmological constant.

b) $\omega(\hat{\phi})=\kappa \hat{\phi}^{m}$ and $\lambda(\hat{\phi})=\sum_{n=1}^{1+2 m} \lambda_{n} \hat{\phi}^{n} ; \kappa>0$ and $\lambda_{1+2 m}<0$ with $m \in \mathcal{Z}$ In this case we get

$$
\Omega=\left(\frac{-m \psi}{\sqrt{8 \kappa}}\right)^{\frac{1}{m}}
$$

and the potential is

$$
V(\psi)=-2 \sum_{n=1}^{1+2 m} \lambda_{n}\left(\frac{-m \psi}{\sqrt{8 \kappa}}\right)^{\frac{2(n-1)}{m}}
$$

we notice that the effective potential is a polynomial and that the highest power is four, this corresponds to the chaotic inflationary scenario studied by Linde [10]. So, provided the initial conditions are not that the field sits motionless at the bottom of the potential we expect inflation in these models (see Moss and Sahni in [9]).

\section{Conclusions}

We have analyzed the general Scalar-Tensor Theory trying to determined under which conditions these will have an inflationary period. By means of a conformal transformation we have shown that in general STT that have non-minimal coupling between the scalar 
field and gravity can be rewritten so as to obtain a minimally coupled theory. The advantage of this is clear. The dynamics of the scalar field can be understood by looking at the potential since the gravitational interaction, at least in the homogeneous cases, reduces to having a damping term in the equation of motion for the scalar field.

Once we have achieved this transformation we can make use of the machinery provided by the No Hair Theorem [9] and the energy conditions to determine if there is inflation. We have done this in two specific cases, and have shown that for all open or flat anisotropic models inflation takes place .

The main attractive of these theories is the fact that the scalar field forms and integral part of the theory, it has a physical meaning and does not have to be postulated ad hoc as in the standard inflationary models. Of course there is still a lot of arbitrariness in these theories, however, in principle it is possible to determine the value and form of the arbitrary functions from observations. In a way the theory is falsifiable by observations. Furthermore, the introduction of the scalar field is motivated by some sort of fundamental principle related to the constancy of the aforementioned ratios. .This, of course, remains to be seen.

Acknowledgments : JSS would like to thank Dr. Octavio Obregon and the Gravitation and Astrophysics Group at the Universidad Autonoma Metropolitan Iztapalapa in Mexico City, Mexico for their hospitality while this work was done. This work was supported in part by DOE and NASA at Fermilab and CONACYT PCEXCNA- 031113 in Mexico. 


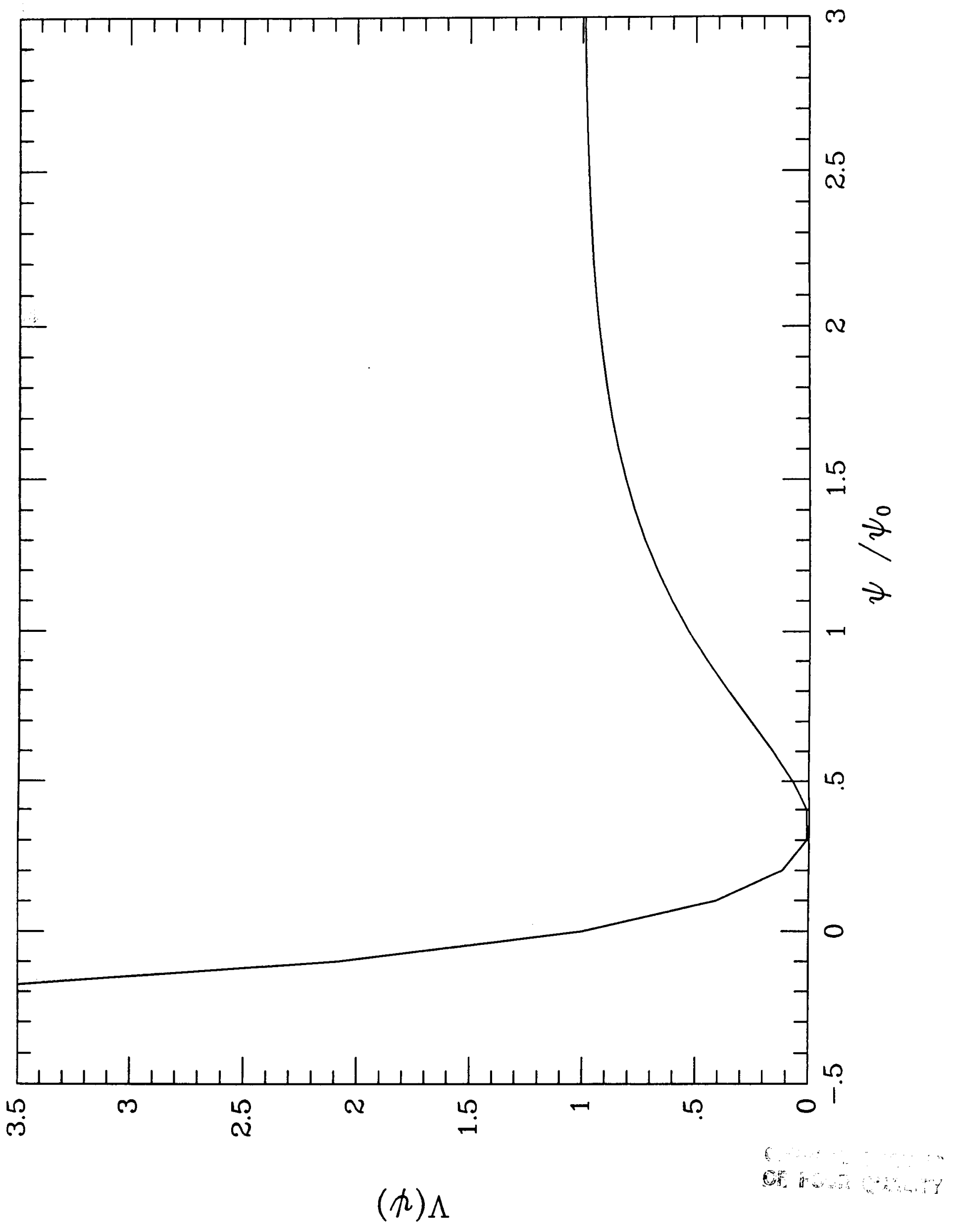


ORIGINR! PRGE IS

OF POOR QUALITY

\section{Figure Caption}

Fig. 1.- The Potential $V(\psi)=1-4 e^{-2 \psi / \psi_{0}}+4 e^{-4 \psi / \psi_{0}}$ with $\psi_{0} \equiv \sqrt{8 \omega_{0}}$ 


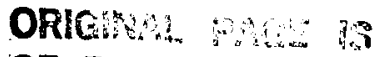

OF. POOR Qunity

\section{References}

1. P.A.M. Dirac, Proc.R.Soc. A165, 199 (1938).

2. P.A.M. Dirac, Proc.R.Soc. A338, 199 (1974).

3. P. Jordan, Astron. Nachr. 276, 193 (1938).

4. S.Weinberg, Gravitation and Cosmology, John Wiley and Sons, (1972).

5. C. Brans and R.H. Dicke, Phys. Rev. 124, 925 (1961).

P.G. Bergmann, Int.J.Theor.Phys. 1,25 (1968).

R.V. Wagoner, Phys. Rev. D1, 3209 (1970).

C.M. Will, Theory and Experiment in Gravitational Physics, CUP, (1981).

T. Singh and L.N. Rai, Gen. Rel. Grav. 15, 875 (1983).

T. Singh and T. Singh, Inter. Jour. Math. Phys. A2, 645 (1987).

6. L.F. Abbott and So-Young Pi, Inflationary Cosmology, World Scientific, Singapore (1986).

7. C. Mathiazhagan and V.B. Jori, Class. Quantum Grav. 1, L39 (1984)

L.O.Pimentel, Astrophys. Space Sci. 112, 175 (1985).

L.O.Pimentel, Astrophys. Space Sci. 116, 395 (1985).

K. Uehara and C.W. Kim, Phys. Rev. D26, 2575 (1982).

8. B. Whitt, Phys. Lett. 145B, 176 (1984).

M. Mijjć and J.A. Stein-Schabes, Phys. Lett. B203, 353 (1988)

Starobinsky and H.-J. Schmidt, Class. Quantum Grav. 4, 695 (1987).

K. Maeda, Phys. Rev. D37, 858 (1988)

J.D. Barrow, Nucl. Phys. B286, 697 (1988)

K. Maeda, J.A. Stein-Schabes and T. Futamase, Fermilab Preprint 88/70-A (1988).

9. R.W. Wald, Phys. Rev. D28, 2118 (1983).

L.G. Jensen and J.A. Stein-Schabes, Phys.Rev.D34, 931 (1986) and D35,1146 (1987).

M.S. Turner and L. Widrow, Phys. Rev. Lett. 57, 2237 (1986).

G. Steigman and M.S. Turner, Phys. Lett 128B, 295 (1983).

I.Moss and V.Sahni, Phys. Lett. 178, 159 (1986).

10. A.D. Linde, Phys. Lett. 129B, 177 (1983). 